\title{
Neuregulin 3 rs10748842 polymorphism contributes to the effect of body mass index on cognitive impairment in patients with schizophrenia
}

Yongjie Zhou ${ }^{1,2}$, Yuhuan $\mathrm{Li}^{3}$, Yujie Meng ${ }^{3}$, Jiesi Wang ${ }^{4}$, Fengchun Wu ${ }^{5}$, Yuping Ning ${ }^{5}$, Yi Li $^{1,2}$, Ryan M. Cassidy , Zezhi $\mathrm{Li}^{7}$ and Xiang Yang Zhang ${ }^{4}$

\begin{abstract}
There is evidence that obesity or higher body mass index is correlated with cognitive impairment in schizophrenia. Recent studies have demonstrated that genetic risk factors, such as the NRG3, are correlated with both elevated BMI and reduced cognitive function. In present study, we aimed to determine whether possession of the NRG3 rs 10748842 influences the correlation between elevated BMI and reduced cognitive ability in schizophrenia. To our knowledge, this has never been examined before. A total of 625 inpatients with schizophrenia and 400 controls were recruited. The Repeatable Battery for the Assessment of Neuropsychological Status (RBANS) was performed to assess cognitive function. We used multiple analysis of covariance (MANCOVA), analyses of covariance (ANCOVA), Pearson correlations, partial correlations, and multivariate regression analysis to test the influence of NRG3 rs10748842 on the aforementioned variables. All RBANS five sub-scores and total score were lower in patients than those in controls (all $p<0.001$ ). Patients carrying NRG3 rs 10748842 TC $+C C$ heterozygous genotype had lower attention score compared to $\Pi$ homozygous genotype (adjusted $F=4.77, p=0.029$ ). BMI was positively associated with language score in patients $(\beta=0.387, t=2.59, p=0.01)$. Interestingly, we further found positive association between BMI and language score in $\Pi$ carriers (partial correlations: $r=0.13$, adjusted $p=0.004$; multivariate regression: $\beta=0.42, t=2.66, p=0.008$ ), but not in CT + CC carrier $(p>0.05)$. Our study demonstrated that NRG3 rs 10748842 was associated with cognitive impairments, especially attention performance in schizophrenia. Moreover, NRG3 rs 10748842 altered the effect of BMI on cognitive impairments as measured by the RBANS language score in chronic patients with schizophrenia.
\end{abstract}

\section{Introduction}

Cognitive impairment is a core characteristic of schizophrenia, observable $\sim 98 \%$ of patients across the domains of memory, attention, language, visuospatial, processing speed, learning, and executive function ${ }^{1,2}$. Cognitive impairments are observed in both first episode

\footnotetext{
Correspondence: Zezhi Li (lizezhi1981@aliyun.com) or

Xiang Yang Zhang (zhangxy@psych.ac.cn)

${ }^{1}$ Research Center for Psychological and Health Sciences, China University of Geosciences, Wuhan, China

${ }^{2}$ Affiliated Wuhan Mental Health Center, Tongji Medical College of Huazhong University of Science \& Technology, Wuhan, China
}

Full list of author information is available at the end of the article. and chronic patients with schizophrenia ${ }^{3}$. They are even observable during their prodromal stage and throughout the illness course despite improvements in psychopathological symptoms after pharmacological treatment ${ }^{4}$. Furthermore, cognitive impairment affects treatment outcomes and everyday disability in patients with schizophrenia $^{5,6}$. Thus, it is of interest to see whether there are mechanisms underlying cognitive impairment, which could be intervened upon to improve outcomes-however, these are multifactorial and are as yet unresolved. Genetic variance is one such mechanism and it is well established that certain genes predispose patients to 
cognitive impairment ${ }^{7}$. The heritability range varies in different domains in patients with schizophrenia, including working memory (0.3-0.6), executive function (0.3-0.6), episodic memory (0.3-0.6), and attention $(0.54)^{8}$. Additional evidence has indicated that cognitive impairment often occurs in unaffected relatives of patients with schizophrenia, ${ }^{9,10}$. On the other hand, accumulating research has suggested that non-genetic factors may also affect cognitive impairments in schizophrenia such as lifestyle ${ }^{11}$, vascular risk factors, and metabolic syndrome ${ }^{12,13}$. Obesity and higher body mass index (BMI) are common in patients with schizophrenia ${ }^{14}$. Indeed even outside of schizophrenia, some studies have shown bidirectional effect of BMI on cognitive impairments ${ }^{15}$. Only a few studies have found no association between BMI and cognitive impairments ${ }^{16,17}$. However, the directional relationship has not been established or the effect of genetic risk factors on this relationship. Nor is it known whether schizophrenia is a modifying influence on the relationship between cognition and BMI.

This question is of interest, because multiple studies have demonstrated a role for genetic factors in both cognitive performance and BMI, showing a common genetic effect on BMI and cognitive function ${ }^{18-20}$. For example, Marioni et al. ${ }^{20}$ found that common genetic variants associated with BMI explain a significant proportion of cognitive function variances and vice versa, indicating shared genetic underpinnings for BMI and cognitive function. Frazier-Wood et al. ${ }^{21}$ demonstrated significant shared genetic effects between cognitive performance and BMI in 1,312 twins. Laitala et al. ${ }^{22}$ provided the evidence of the genetic effects on the relationship between midlife BMI and senile cognition in a large sample of 2606 Finnish twins. Thus, this interaction seems like a promising avenue of exploration for both the etiology and therapy of obesity and cognitive impairment. However, to our knowledge, no study has investigated whether genetic factor, BMI and their interactions might affect cognitive function in schizophrenia, and the BMI might influence cognitive performance based on different genetic background.

A prominent hypothesis on the etiology of cognitive impairment and schizophrenia is neurodevelopmental dysfunction, leading to altered basic neurocircuitry ${ }^{23,24}$. Neuregulin 3 (NRG3), a neuronal enriched growth factor, plays an important role in neuronal development, including plasticity, development, differentiation, and proliferation ${ }^{25}$. NRG3 is located on chromosome 10q22q23; variation in this locus predisposes individuals to schizophrenia as demonstrated by genome-wide studies $^{26,27}$. In addition, NRG3 mutation has been indicated to affect neurocognitive impairment ${ }^{28}$, especially in patients with schizophrenia ${ }^{29}$.
Strikingly, NRG3 has also been implicated to be associated with BMI in an Asian population through genomewide scanning ${ }^{30}$. Therefore, in this study, we sought to determine (1) whether the NRG3 polymorphism rs10748842 might affect cognitive impairment in schizophrenia; (2) whether BMI might affect cognitive impairments in schizophrenia; and (3) whether NRG3 polymorphism rs10748842 mediates the effects of BMI on cognitive impairment in schizophrenia in a large sample of Chinese Han population.

\section{Materials and methods \\ Subjects}

The present study was reviewed and approved by the Institutional Review Boards of Beijing Hui-Long-Guan hospital. Written informed consents were obtained from all subjects or guardians. The patients recruited in this study satisfied the following inclusion criteria: (1) Han Chinese (2) aged from 18 to 75 years; (3) met the criteria of the Diagnostic and Statistical Manual of Mental Disorders, Fourth Edition (DSM-IV) for schizophrenia; (4) had at least 5 years of illness duration; (5) had been treated with antipsychotics for at least 12 months, primarily with one antipsychotic drug.

A total of 625 patients were recruited with an average age of $45.03 \pm 10.19$ years. Antipsychotics included clozapine $(n=273)$, risperidone $(n=114)$, quetiapine $(n=56)$, chlorpromazine $(n=46)$, aripiprazole $(n=32)$, sulpiride $(n=27)$, perphenazine $(n=19)$, olanzapine $(n=17)$, haloperidol $(n=7)$, and others $(n=34)$. The antipsychotic doses equivalent to chlorpromazine were $390.35 \pm 295.35 \mathrm{mg} /$ day. A total of 400 healthy controls were recruited from the local community. Subjects with any major Axis I disorders or with family history of mental disorders were excluded. All subjects underwent physical examinations and lab tests prior to enrollment. Any subjects with cancer, infections, uncontrolled diabetes, cerebrovascular disease, cardiovascular disease, and pregnancy were excluded. Neither patients nor healthy controls had drug or alcohol abuse/dependence other than smoking. The demographic and clinical profile of subjects were shown in Table 1.

\section{Clinical interview and cognitive assessments}

An in-house socio-demographic scale was used to collect relevant information. The Structure Clinical Interview for DSM-IV (SCID-I/P) was used to screen each participant as conducted independently by two psychiatrists. Clinical symptoms of patients were assessed by using the Positive and Negative Syndrome Scale (PANSS) ${ }^{31}$.

Cognitive performance of participants was assessed by using the Repeatable Battery for the Assessment of Neuropsychological Status (RBANS, Form A). We used the translated Chinese version of this test, which has 
Table 1 Demographic profiles in patients with schizophrenia and controls.

\begin{tabular}{|c|c|c|c|c|}
\hline Variable & Patients $(N=625)$ & Controls $(N=400)$ & Statistic & $p$ \\
\hline Age (years) & $45.03 \pm 10.19$ & $44.73 \pm 13.50$ & 0.37 & 0.71 \\
\hline Gender (male) & $512(81.9 \%)$ & 157 (39.3\%) & 159.90 & $<0.0001$ \\
\hline Education (years) & $8.45 \pm 3.65$ & $9.73 \pm 5.60$ & 4.06 & $<0.0001$ \\
\hline BMI & $23.97 \pm 4.45$ & $25.16 \pm 4.26$ & 4.20 & $<0.0001$ \\
\hline Onset age & $26.60 \pm 20.19$ & - & - & - \\
\hline Illness duration (years) & $21.14 \pm 9.90$ & - & - & - \\
\hline Atypical antipsychotic ( $n$ ) & 439 & - & - & - \\
\hline Typical antipsychotic (n) & 186 & - & - & - \\
\hline Daily dose (mg/day) (chlorpromazine equivalents) & $390.35 \pm 295.35$ & - & - & - \\
\hline Antipsychotic treatment duration (months) & $31.37 \pm 46.64$ & - & - & - \\
\hline PANSS score & & - & - & - \\
\hline Positive symptoms & $11.92 \pm 6.26$ & - & - & - \\
\hline Negative symptoms & $20.14 \pm 8.69$ & - & - & - \\
\hline General psychopathology & $25.15 \pm 7.11$ & - & - & - \\
\hline Total score & $57.20 \pm 16.51$ & - & - & - \\
\hline
\end{tabular}

established validity and test-retest reliability in patients with schizophrenia and healthy controls ${ }^{32}$. The RBANS assesses cognitive impairment via a total score and scores for immediate memory, attention, language, visuospatial/ constructional, and delayed memory, calculated by ageadjusted index scores through 12 subscales. All patients were in stable state without acute psychotic symptoms ${ }^{32}$.

\section{DNA extraction and SNP genotyping}

DNA was obtained from the peripheral vein blood of each participant through salting-out method and stored in -80 degree $^{31}$. The NRG3 polymorphism rs10748842 was genotyped by using Matrix-Assisted Laser Desorption/Ionization Time of Flight Mass Spectrometry (MALDI-TOF MS) in the MassARRAY System (Sequenom Inc., San Diego, CA, USA) according to the method described in our previous studies $^{31}$. A randomly selected $5 \%$ of samples were repeatgenotyped for quality control; the error rate was $0.1 \%$.

\section{Statistical analyses}

Student's $t$-test and Chi-square test were used for continuous variables and categorical variables, respectively. The $x^{2}$ test for goodness of fit was used for Hardy-Weinberg equilibrium in patients and healthy control subjects. Since there was almost no homozygous variant CC genotype in our study (about $0.96 \%$ in the patients and $0.25 \%$ in healthy controls), we combined the $\mathrm{CC}$ and TC genotypes as a group.

To detect the difference in cognitive scores between patients and controls, multiple analysis of covariance
(MANCOVA) was applied to investigate the association between genotype and cognition. We set cognition scores as dependent variables, with diagnosis as the fixed factor, and adjusted for sex, BMI, and years of education as covariates. Then the models of covariance (ANCOVA) were applied using the RBANS five subset and total scores as the dependent variables respectively, with the diagnosis as an independent variable, and with sex, years of education, BMI, and smoking as covariates.

To identify the differences in cognitive scores based on different genotypes in the patients or controls, the model of $2 \times 2$ MANCOVA (genotype $\times$ diagnosis) was first used to report the overall $\mathrm{p}$ value, and then in this model the main effects of diagnosis, genotype, and genotype $\times$ diagnosis were tested. In this MANCOVA model, the diagnosis and the NRG3 polymorphism rs10748842 genotype were used as the independent variables, with sex, BMI, years of education, and smoking as the covariates. Furthermore, the main effects of diagnosis, genotype, and genotype $\times$ diagnosis were also examined through MANCOVA model.

Then, ANCOVA analysis was applied, with age, sex, BMI, education, smoking, as the covariates in control group, together with illness duration, hospitalization times, onset age, antipsychotic types (typical antipsychotics or atypical antipsychotics), daily dose, and antipsychotic duration as covariates in patients. Bonferroni corrections were used in each test for multiple tests. Further, we used multivariate regression analysis (stepwise) with the RBANS five subset and total scores as 
Table 2 Demographic profiles in different genotypic groups among patients and controls.

\begin{tabular}{|c|c|c|c|c|c|c|c|c|}
\hline \multirow[t]{2}{*}{ Variable } & \multicolumn{4}{|c|}{ Patients $(N=625)$} & \multicolumn{4}{|c|}{ Controls $(N=400)$} \\
\hline & $\begin{array}{l}\text { TT } \\
(N=544)\end{array}$ & $\begin{array}{l}\mathrm{TC} \\
(N=81)\end{array}$ & Statistic & $p$ & $\begin{array}{l}\text { TT } \\
(N=347)\end{array}$ & $\begin{array}{l}\mathrm{TC} \\
(N=53)\end{array}$ & Statistic & $p$ \\
\hline Age (years) & $44.91 \pm 10.21$ & $45.84 \pm 10.81$ & 0.77 & 0.44 & $44.67 \pm 13.53$ & $45.15 \pm 13.44$ & 0.24 & 0.81 \\
\hline Gender (male) & $447(82.2 \%)$ & 66 (81.5\%) & 0.17 & 0.92 & 135 (38.9\%) & $22(41.5 \%)$ & 0.13 & 0.72 \\
\hline Education (years) & $8.42 \pm 3.75$ & $8.70 \pm 2.93$ & 0.67 & 0.50 & $9.94 \pm 5.80$ & $8.38 \pm 3.86$ & 1.90 & 0.06 \\
\hline BMl & $23.97 \pm 4.52$ & $23.99 \pm 4.00$ & 0.04 & 0.97 & $25.10 \pm 4.20$ & $25.50 \pm 4.64$ & 0.62 & 0.53 \\
\hline Onset age & $23.76 \pm 6.80$ & $30.21 \pm 23.17$ & 1.01 & 0.28 & - & - & - & - \\
\hline Illness duration (years) & $21.13 \pm 9.95$ & $21.19 \pm 9.64$ & 0.05 & 0.96 & - & - & - & - \\
\hline Daily dose (mg/day) (chlorpromazine equivalents) & $390.47 \pm 308.32$ & $389.49 \pm 187.59$ & 0.03 & 0.98 & - & - & - & - \\
\hline Antipsychotic treatment duration (months) & $31.27 \pm 47.27$ & $32.10 \pm 42.52$ & 0.15 & 0.88 & - & - & - & - \\
\hline \multicolumn{9}{|l|}{ PANSS score } \\
\hline Positive symptoms & $11.95 \pm 6.36$ & $11.67 \pm 5.54$ & 0.37 & 0.71 & - & - & - & - \\
\hline Negative symptoms & $19.94 \pm 8.62$ & $21.48 \pm 9.11$ & 1.48 & 0.14 & - & - & - & - \\
\hline General psychopathology & $25.10 \pm 7.11$ & $25.52 \pm 7.19$ & 0.49 & 0.62 & - & - & - & - \\
\hline Total score & $56.99 \pm 16.56$ & $58.67 \pm 16.17$ & 0.85 & 0.40 & - & - & - & - \\
\hline
\end{tabular}

dependent variables respectively, using NGR3 genotype as an independent variable in the patients, adjusting for covariates, including age, sex, BMI, education, smoking, illness duration, onset age, antipsychotic types, daily dose, and current antipsychotic medication duration in patients. ANCOVA was also used to identify the main effect of the NRG3 genotype on BMI.

To investigate the effects of BMI on cognitive performance in patient and control groups, multivariate regression was applied, with the RBANS five subset and total scores as dependent variables, using BMI as an independent variable, adjusting for demographic and clinical covariates in patients and controls, respectively.

Pearson correlations were used to examine whether cognitive function was related to BMI in different genotype groupings in the patient group. Partial correlations were used adjusting for age, sex, years of education, smoking, illness duration, onset age, antipsychotic types, daily dose, and antipsychotic duration. Further, in each NGR3 genotype group, multivariate regression analysis (stepwise) was used with the RBANS five subsets and total score as dependent variables, using BMI as independent variables, adjusting for covariates including age, sex, education, smoking, illness duration, onset age, antipsychotic types, daily dose antipsychotic duration in patients.

Quanto Software was used to calculate the power of the sample under log additive, recessive and dominant models, setting $1 \%$ as the prevalence of schizophrenia in population.

\section{Results}

The demographic and clinical profiles of the patients and healthy controls were detailed in Table 1. Except for age, significant differences in sex, BMI and years of education were found between patients and healthy controls (all $p<0.0001$ ), adjusted as covariates in the following analyses. Table 2 shows the demographic and clinical data by NRG3 rs10748842 genotype in case and control groups, respectively.

\section{Association analysis of NRG3 rs 10748842 with schizophrenia}

Genotyping was completed in 625 patients and 400 controls. The Hardy-Weinberg equilibrium of NRG3 rs10748842 genotypes were consistent in both patient and control groups (both $p>0.05$ ). The distributions of NRG3 rs10748842 allele and genotype were not significantly different between patient and control groups $\left(\chi^{2}=0.09\right.$, $p=0.77 ; \chi^{2}=1.91, p=0.39$; respectively). Further logistic regression analysis (backward conditional) demonstrated no significant difference in the distribution of NRG3 rs10748842 allele or genotype between patient and control groups (both $p>0.05$ ).

\section{Cognition between patients and controls}

As predicted, MANCOVA showed significant difference in cognitive perform between patient and control groups (Wilks' lambda $F=112.06 ; p<0.001$ ). Then ANCOVA analysis conducted for each cognitive score showed that all RBANS scores were significantly decreased in patients 
Table 3 Comparisons among the RBANS total and five domain scores by diagnostic and genotypic groups.

\begin{tabular}{|c|c|c|c|c|c|c|c|c|c|c|}
\hline \multirow[t]{2}{*}{ RBANS scores } & \multicolumn{2}{|c|}{ Patients with schizophrenia ${ }^{a}$} & \multicolumn{2}{|l|}{ Controls } & \multicolumn{2}{|c|}{ Diagnosis } & \multicolumn{2}{|c|}{ Genotype } & \multicolumn{2}{|c|}{$\begin{array}{l}\text { Diagnosis X } \\
\text { genotype }\end{array}$} \\
\hline & $\begin{array}{l}\mathrm{TT} \\
(N=544)\end{array}$ & $\begin{array}{l}\mathrm{TC}+\mathrm{CC} \\
(N=81)\end{array}$ & $\begin{array}{l}\mathrm{TT} \\
(N=347)\end{array}$ & $\begin{array}{l}\mathrm{TC}+\mathrm{CC} \\
(N=53)\end{array}$ & $F$ & $p$ & $F$ & $p$ & $F$ & $p$ \\
\hline Immediate memory & $56.28 \pm 15.10$ & $54.24 \pm 15.65$ & $76.02 \pm 17.07$ & $74.06 \pm 20.33$ & 167.34 & $<0.001$ & 1.71 & 0.19 & 0.001 & 0.98 \\
\hline Attention & $61.83 \pm 17.70^{\mathrm{a}}$ & $57.70 \pm 13.08^{\mathrm{a}}$ & $87.87 \pm 20.26$ & $84.47 \pm 21.43$ & 226.60 & $<0.001$ & 4.60 & $0.03^{b}$ & 0.04 & 0.87 \\
\hline Language & $73.42 \pm 17.54$ & $70.54 \pm 16.70$ & $94.15 \pm 12.98$ & $92.85 \pm 13.96$ & 203.97 & $<0.001$ & 1.92 & 0.17 & 0.28 & 0.60 \\
\hline Visuospatial/construction & $75.02 \pm 17.50$ & $71.70 \pm 15.94$ & $80.08 \pm 15.89$ & $78.06 \pm 13.73$ & 13.09 & $<0.001$ & 2.88 & 0.09 & 0.18 & 0.68 \\
\hline Delayed memory & $61.99 \pm 18.37$ & $61.96 \pm 18.09$ & $86.54 \pm 14.93$ & $85.06 \pm 17.48$ & 213.15 & 0.001 & 0.21 & 0.64 & 0.20 & 0.66 \\
\hline Total score & $59.12 \pm 13.38$ & $56.64 \pm 11.35$ & $80.41 \pm 15.02$ & $78.30 \pm 16.70$ & 261.71 & $<0.001$ & 2.98 & 0.08 & 0.02 & 0.89 \\
\hline BMI & $23.97 \pm 4.52$ & $23.99 \pm 4.00$ & $25.10 \pm 4.20$ & $25.50 \pm 4.64$ & 10.00 & 0.002 & 0.25 & 0.62 & 0.20 & 0.66 \\
\hline
\end{tabular}

${ }^{a}$ A significant genotypic effect on Attention score in patients with schizophrenia.

${ }^{\mathrm{b}}$ There was a significant genotype effect on Attention score in all subjects $(F=4.60, p=0.03)$. Patients carrying TC $+C C$ had lower attention score than those carrying $C C$ in patient group (adjusted $F=4.77, p=0.029$ ). There was no difference in attention score between controls carrying TC $+C C$ and carrying $C C$ (adjusted $F=0.20, p=0.66$ ).

compare to healthy controls (all $p<0.001$ ) (Table 3). All these significant differences survived after Bonferroni correction (all $p<0.001)$.

\section{Effects of NRG3 rs 10748842 on cognition in patients and controls}

As predicted, MANCOVA analysis showed significant main effect of diagnosis (Wilks' lambda $F=61.54$; $p<0.001$ ), but no effect of either genotype (Wilks' lambda $F=0.87 ; p=0.52$ ) or genotype $\mathrm{x}$ diagnosis (Wilks' lambda $F=0.32 ; p=0.93)$. Then ANCOVA analysis was conducted for each cognitive score shown in Table 3, and a significant genotype effect on attention score was found in all subjects $(F=4.60, p=0.03)$. Further, ANCOVA analysis demonstrated that patients carrying $\mathrm{TC}+\mathrm{CC}$ had lower attention score than those carrying TT only in patient group $(F=4.07, p=0.04)$. After adjusting for the age, sex, education years, smoking, illness duration, onset age, medication type, dose, and medication duration as the covariates, the difference still remained significant (adjusted $F=4.77, p=0.029)$. However, no difference was found in attention score between TC $+\mathrm{CC}$ carrier and TT carrier in control group (adjusted $F=0.20, p=0.66$ ). We did not find significant genotype $\mathrm{x}$ diagnosis effects on other RBANS scores and total score (all $p>0.05$ ) (Table 3).

Multivariate stepwise regression further showed that the genotype of NRG3 rs10748842 was correlated with the attention score independently only in patient group $(\beta=-4.40, t=2.27, p=0.02)$.

\section{Effects of NRG3 rs10748842 on BMI in patients and controls}

There was a significant main effect of diagnosis on BMI $(F=10.00, p=0.002)$ (Table 3$)$. No genotype or diagnosis $\times$ genotype effects on BMI were observed (all $p>0.05)$. As shown in Table 3, there was no difference in BMI between genotypes in the all subjects or when the patients and healthy controls were detected separately (all $p>0.05$ ). It suggested that NRG3 rs10748842 genotype had no effect on BMI in patients with schizophrenia.

\section{Correlation of BMI with cognitive performance in patients and controls}

Multivariate regression showed that BMI was positively associated with language $(\beta=0.387, t=2.59, p=0.01)$ in patients, but not with other RBANS subscales or total score. There was no association of BMI with any RBANS subscale or total scores (all $p>0.05$ ).

\section{Relationships between BMI and cognitive score: affected by NRG3 rs10748842}

We further explored whether the relationship of BMI with cognitive function differed between genotypic subgroups in patients and healthy controls separately. In patient group, Pearson correlations showed significant positive association between BMI and language score in TT carriers $(r=0.14, p=0.001)$. After adjusting for age, sex, years of education, smoking, illness duration, onset age, antipsychotics type, antipsychotics dose (chlorpromazine equivalents), and antipsychotics treatment duration, partial correlation analysis showed positive association between BMI and language score in patients with TT genotype $(r=$ $0.13, p=0.004$ ) (Fig. 1). Further, multivariate regression confirmed that BMI was positively associated with language $(\beta=0.42, t=2.66, p=0.008)$ in patients carrying TT genotype. However, there was no association between BMI and language performance in patients carrying $\mathrm{TC}+\mathrm{CC}$ genotype, adjusting for clinical covariates $(r=0.02, p=0.83)$. 


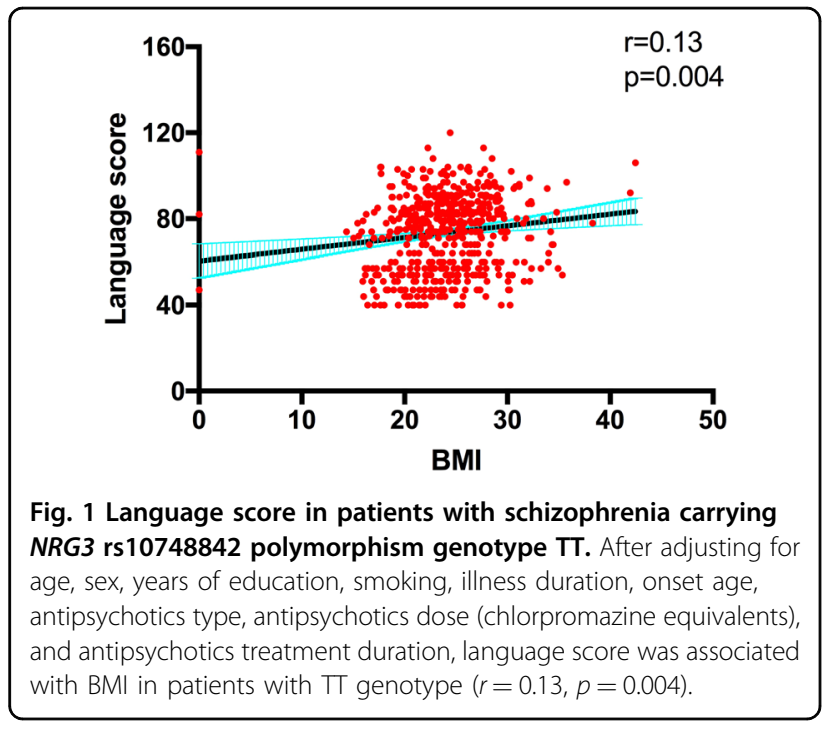

In control group, there was no association between BMI and any RBANS subscales, as well as total score in each different NGR3 genotypic subgroup (all $p>0.05$ ), suggesting that there was no significant interaction effect of BMI*NGR3 genotype on cognitive function in the control group.

\section{Discussion}

In the present study, we found that (1) all RBANS scores were decreased in patients with schizophrenia compared to healthy controls; (2) patients with NRG3 rs10748842 genotype TC $+\mathrm{CC}$ had lower attention score than those with TT; (3) BMI was positively associated with the language score in schizophrenia. Interestingly, when stratified by genotype, positive association between BMI and language score was found only in patients with TT genotype.

Cognitive impairment is common in the patients with schizophrenia. Our results were consistent with previous study showing worse cognitive function in patients with schizophrenia than in healthy controls ${ }^{33}$. However, up to date, the underlying mechanisms of cognitive deficits in schizophrenia are unclear. Some studies have indicated that genetic factors are involved in cognitive impairment in schizophrenia ${ }^{7}$. The neurodevelopmental hypothesis has been proposed for the etiology and cognitive impairment in schizophrenia ${ }^{34,35}$. Neuregulin 3 (NRG3) is a member of neuregulin family, which plays an important role in neuronal development, including plasticity, development, differentiation, and proliferation ${ }^{25}$. Previous evidence indicated that NRG3 gene mutations were correlated with cognitive impairment in patients with schizophrenia $^{29,36,37}$. Further, some of these studies also demonstrated the specific association or opposite association between NRG3 gene mutations and cognitive inpatients in schizophrenia and healthy controls. For example, Morar et al. ${ }^{29}$ found that NRG3 mutation might adjust early attentional processes with opposite influence between patients with schizophrenia and healthy controls. Tost et al. ${ }^{37}$ further provided the evidence indicating that NRG3 polymorphism rs10748842 affected neuronal function in the prefrontal cortex in schizophrenia patients, but this effect was not found in healthy subjects or in healthy siblings of patients. Recent study also showed that NRG3 rs10748842 was associated with cognitive impairment only in schizophrenia ${ }^{38}$. However, the underlying mechanism of this patient-specific association might be complex and has not been investigated. We speculated that the following aspects might interpret this phenomenon. First, previous evidence has demonstrated that NRG3 can regulate dopaminergic neurotransmis$\operatorname{sion}^{39,40}$. Further, the activation of Neuregulin-ErbB signaling pathway can also mediate the dopamine levels ${ }^{41}$. Second, Bartolini et al. ${ }^{42}$ proved that NRG3 could regulate the final allocation of GABAergic interneurons in the cortex. It has been established that the dysfunction of dopaminergic neurotransmission and GABAergic neurotransmission has been believed to be involved in pathophsiology of schizophrenia ${ }^{43,44}$. On the other hand, dopamine and GABAergic neurotransmission can play a critical role in cognitive functions ${ }^{45,46}$. Thus, taken together, the abnormality of NGR3 might enlarge the dysfunction of dopaminergic neurotransmission and GABAergic neurotransmission in patients with schizophrenia, which can cause more significant cognitive impairment in schizophrenia than in healthy controls.

In the present study, we provided further evidence of genetic effects on cognitive impairment, showing that NRG3 polymorphism rs10748842 was associated with cognitive impairment, especially attention performance in schizophrenia. NRG3 polymorphism rs10748842 is located in the non-exonic ultra-conserved genomic elements (UCEs), where critical functional element TAATTA motif exists, and the rs10748842 (T) positively binds with multiple important family of transcript factors, and thus rs10748842 strongly affects and predicts NRG3 expression in human brain ${ }^{47,48}$. In addition, rs10748842 can affect NRG3 class II and class III, specifically expressed in brain ${ }^{49}$. Kao et al. ${ }^{48}$ demonstrated that NRG3 expression in patients with schizophrenia carrying rs10748842 C allele was $60 \%$ lower than those in patients carrying the $\mathrm{T}$ allele. Paterson et al. ${ }^{49}$ also observed in both bipolar disorder and depression that NRG3 levels of isoforms II and III were lower in patients with heterozygous TC genotype than those in $\mathrm{T}$ allele carriers.

The impact of BMI on cognitive function is a public health problem in adolescence and adulthood, but the results are still inconsistent. Recently Olivo et al. ${ }^{50}$ demonstrated worse cognitive function in overweight and 
obese individuals from the UK Biobank cohort with a sample of 170,310 participants. However, Qizilbash et al. $^{51}$ reported that lower BMI in middle-aged and elderly people was found to have higher risk of dementia in cohort of 1,958,191 people. Similarly, till now, the high prevalence of metabolic syndrome-especially obesity and higher BMI-in patients with schizophrenia has also received increasing attention. The relationship between BMI and cognitive impairment in schizophrenia has been recognized, but the results were also inconclusive ${ }^{12}$. Some studies have shown poor cognitive performance in schizophrenia patients with higher BMI. For example, Kimhy et al $^{52}$ showed that BMI was inversely associated with cognitive performance. Rashid et al. ${ }^{13}$ indicated that BMI could be used as a potential predictor of higher risk of cognitive impairment in schizophrenia patients. Hidese et al. ${ }^{53}$ recently reported that BMI was negatively associated with cognitive performance in schizophrenia patients. Friedman et al. ${ }^{16}$ demonstrated that a BMI above $25 \mathrm{~kg} / \mathrm{m}^{2}$ produced negative effects on cognition in both patient group and control group ${ }^{16}$. However, some studies showed no association between BMI and cognition. For example, Takayanagi et al. ${ }^{17}$ found no assocaitation between BMI and cognitive impairment in samples of 1289 individuals with schizophrnia ${ }^{16}$. In addition, some studies have shown a bidirectional effect of BMI on cognitive impairment ${ }^{15}$. Taken together, the abovementioned findings indicate that the effects of BMI on cognitive impairment in schizophrenia and general population are far more complicated than expected. Other factors might contribute to the impacts of BMI on cognitive impairments in patients with schizophrenia.

Lines of evidence shows shared genetic effects on BMI and cognitive function, and genetic correlation quantified the genetic variants for BMI associated with genetic variants for cognitive function ${ }^{20}$. Relative genes associated with both cognitive function and BMI may have overlapping expression in the anatomical parts of the brain ${ }^{19}$. For example, Marioni et al. ${ }^{20}$ reported that genetic variants associated with better cognitive performance were associated with lower BMI. Frazier-Wood et al. ${ }^{21}$ demonstrated around $30 \%$ of the genetic correlation between cognitive performance and $\mathrm{BMI}^{21}$. Laitala et al. ${ }^{22}$ showed $12 \%$ additive genetic correlation between cognitive performance in the elderly and midlife BMI. Taken together, the above findings suggest shared genetic pathway for BMI and cognitive function. Interestingly, NRG3 gene was reported to be associated with BMI through genome-wide association study in Asian population $^{30}$. Thus, we reasonably investigated whether BMI affected cognitive impairment in schizophrenia was associated with NRG3 polymorphism rs10748842. We found that BMI was positively associated with language score in patients with schizophrenia. More interestingly, when performing genotypic stratification analysis, we found a positive association between BMI and language score only in TT patients, but not in CT + CC genotypes, indicating that the interaction between NRG3 and BMI might affect cognitive function in schizophrenia. It is likely that the BMI might influence cognitive performance based on different NRG3 genotypes. Previous findings on the impact of BMI on cognitive impairment were inconsistent, probably because they did not consider other interactions, such as genetic factors. To our best knowledge, the current study is the first to provide evidence for the interaction of gene and BMI in cognitive impairment of schizophrenia.

However, the underpinning of the correlation between NRG3 gene and BMI have been suggested. Guénard et al. ${ }^{54}$ found that the NRG3 gene associated with obesity traits was correlated with dietary pattern, suggesting that it was involved in metabolic responses to dietary patterns of obesity and glucose metabolism. Meanwhile, the association between BMI and cognition has also been implicated. Some studies indicated that the neurochemical mediators induced by adipocyte could regulate neuronal function directly ${ }^{55}$. On the other hand, leptin predominantly induced by adipocytes could affect appetite and contribute to cognition ${ }^{56}$. Additionally, higher leptin levels have been reported to be correlated with higher $\mathrm{BMI}^{57}$, and leptin could enhance synaptic long-term potentiation and plasticity ${ }^{58,59}$. However, further mechanism on interplay of NRG3 and BMI in cognitive impairments should be investigated in future.

There were some limitations that should be concerned to carefully interpret our results. First, the patients recruited in the present study had chronic schizophrenia, thus it could not represent this influence in first episode patients. However, some studies have indicated that cognitive impairment did not change within the same patient over time, and they could remain relatively stable. Further, cognitive impairment was independent of changes in clinical status, and the pattern and severity of cognitive impairment were generally stable in different clinical conditions of patients ${ }^{60}$. Second, the patients recruited in present study were treated with different antipsychotics, which may affect cognitive function, although they were equivalently converted to chlorpromazine and controlled in the statistical analyses. Third, the results of our study might not be interpreted in other ethical population. Last but not the least, the association of NG3 rs10748842 with cognition was only marginal, and the effect was only seen in patient group. Thus, there is a need to replicate these findings in additional independent sample, including different situation or stages of the patients. For example, first episode patients, drug naive patients or patients treated with one certain antipsychotic medicine, or other ethical populations. 
In conclusion, our study demonstrated that genetic factor NRG3 rs10748842 might be associated with cognitive impairment, especially attention performance in schizophrenia. Further, BMI might affect cognitive function under certain NRG3 rs10748842 genotype in schizophrenia, suggesting that NRG3 rs10748842 might contribute to the effects of BMI on cognitive impairment in chronic patient with schizophrenia.

\section{Acknowledgements}

We would like to thank Dr. Dachun Chen, Mei Hong Xiu, Humei An, Song Chen, and Gui Gang Yang for all of their hard work and significant contributions toward the study. This study was supported by the National Natural Science Foundation of China (81371477; 81401127), Shanghai Jiao Tong University Medical Engineering Foundation (YG2016MS48), Excellent talent foundation of Shanghai Jiao Tong University School of Medicine (19XJ1 1006), the Science and Technology Program of Guangzhou (201807010064), and project of Wuhan Municipal Health and Family planning Commission (WX15C06, WG12A0). All funding for this study had no further role in study design, data analysis, and in the decision to submit the paper for publication.

\section{Author details}

${ }^{1}$ Research Center for Psychological and Health Sciences, China University of Geosciences, Wuhan, China. ${ }^{2}$ Affiliated Wuhan Mental Health Center, Tongji Medical College of Huazhong University of Science \& Technology, Wuhan, China. ${ }^{3}$ Qingdao Mental Health Center, Qingdao, China. ${ }^{4}$ CAS Key Laboratory of Mental Health, Institute of Psychology, Chinese Academy of Sciences, Beijing, China. ${ }^{5}$ The Affiliated Brain Hospital of Guangzhou Medical University (Guangzhou Huiai Hospital), Guangzhou, China. ${ }^{6}$ Department of Psychiatry and Behavioral Sciences, The University of Texas Health Science Center at Houston, Houston, TX, USA. ${ }^{7}$ Department of Neurology, Ren Ji Hospital, Shanghai Jiao Tong University School of Medicine, Shanghai, China

\section{Data availability}

All the data are available by requirement from the corresponding author.

\section{Conflict of interest}

The authors declare that they have no conflict of interest.

\section{Publisher's note}

Springer Nature remains neutral with regard to jurisdictional claims in published maps and institutional affiliations.

Received: 7 September 2019 Revised: 7 December 2019 Accepted: 19 December 2019

Published online: 11 February 2020

\section{References}

1. Keefe, R. S., Eesley, C. E. \& Poe, M. P. Defining a cognitive function decrement in schizophrenia. Biol. Psychiatry 57, 688-691 (2005).

2. Tripathi, A., Kar, S. K. \& Shukla, R. Cognitive deficits in schizophrenia: understanding the biological correlates and remediation strategies. Clin. Psychopharmacol. Neurosci. 16, 7-17 (2018).

3. Gerretsen, P. et al. Insight into illness and cognition in schizophrenia in earlier and later life. J. Clin. Psychiatry 78, e390-e397 (2017).

4. Kahn, R. S. \& Keefe, R. S. Schizophrenia is a cognitive illness: time for a change in focus. JAMA Psychiatry 70, 1107-1112 (2013).

5. Bowie, C. R. et al. Prediction of real-world functional disability in chronic mental disorders: a comparison of schizophrenia and bipolar disorder. Am. J. Psychiatry 167, 1116-1124 (2010).

6. Harvey, P. D., Keefe, R. S., Patterson, T. L., Heaton, R. K. \& Bowie, C. R. Abbreviated neuropsychological assessment in schizophrenia: prediction of different aspects of outcome. J. Clin. Exp. Neuropsychol. 31, 462-471 (2009).
7. Toulopoulou, T. et al. Substantial genetic overlap between neurocognition and schizophrenia: genetic modeling in twin samples. Arch. Gen. Psychiatry 64, 1348-1355 (2007).

8. Harvey, P. D. et al. Factor structure of cognition and functional capacity in two studies of schizophrenia and bipolar disorder: implications for genomic studies. Neuropsychology 30, 28-39 (2016).

9. Snitz, B. E., Macdonald, A. W. 3rd \& Carter, C. S. Cognitive deficits in unaffected first-degree relatives of schizophrenia patients: a meta-analytic review of putative endophenotypes. Schizophr. Bull. 32, 179-194 (2006).

10. Toulopoulou, T. et al. Impaired intellect and memory: a missing link between genetic risk and schizophrenia? Arch. Gen. Psychiatry 67, 905-913 (2010).

11. Vinogradov, S. et al. The cognitive cost of anticholinergic burden: decreased response to cognitive training in schizophrenia. Am. J. Psychiatry 166, 1055-1062 (2009).

12. Bora, E., Akdede, B. B. \& Alptekin, K. The relationship between cognitive impairment in schizophrenia and metabolic syndrome: a systematic review and meta-analysis. Psychol. Med 47, 1030-1040 (2017).

13. Rashid, N. A. et al. Unraveling the relationship between obesity, schizophrenia and cognition. Schizophr. Res. 151, 107-112 (2013).

14. Li, Q. et al. The prevalence, risk factors and clinical correlates of obesity in Chinese patients with schizophrenia. Psychiatry Res. 251, 131-136 (2017).

15. Smith, E., Hay, P., Campbell, L. \& Trollor, J. N. A review of the association between obesity and cognitive function across the lifespan: implications for novel approaches to prevention and treatment. Obes. Rev. 12, 740-755 (2011).

16. Friedman, J. I. et al. The effects of hypertension and body mass index on cognition in schizophrenia. Am. J. Psychiatry 167, 1232-1239 (2010).

17. Takayanagi, Y., Cascella, N. G., Sawa, A. \& Eaton, W. W. Diabetes is associated with lower global cognitive function in schizophrenia. Schizophr. Res. 142, 183-187 (2012).

18. Davies, G. et al. Genetic contributions to variation in general cognitive function: a meta-analysis of genome-wide association studies in the CHARGE consortium ( $N=53949)$. Mol. Psychiatry 20, 183-192 (2015).

19. Locke, A. E. et al. Genetic studies of body mass index yield new insights for obesity biology. Nature 518, 197-206 (2015).

20. Marioni, R. E. et al. Assessing the genetic overlap between BMl and cognitive function. Mol. Psychiatry 21, 1477-1482 (2016).

21. Frazier-Wood, A. C. et al. Cognitive performance and BMI in childhood: shared genetic influences between reaction time but not response inhibition. Obes. (Silver Spring) 22, 2312-2318 (2014).

22. Laitala, V. S. et al. Association and causal relationship of midlife obesity and related metabolic disorders with old age cognition. Curr. Alzheimer Res 8 , 699-706 (2011).

23. Lin, Z. et al. The interaction of BDNF and NTRK2 gene increases the susceptibility of paranoid schizophrenia. PLOS ONE 8, e74264 (2013).

24. Lu, W. et al. Association between BDNF Val66Met polymorphism and cognitive performance in antipsychotic-naive patients with schizophrenia. J. Mol. Neurosci. 47, 505-510 (2012).

25. Carteron, C., Ferrer-Montiel, A. \& Cabedo, H. Characterization of a neuralspecific splicing form of the human neuregulin 3 gene involved in oligodendrocyte survival. J. Cell Sci. 119, 898-909 (2006).

26. Fallin, M. D. et al. Genomewide linkage scan for schizophrenia susceptibility loci among Ashkenazi Jewish families shows evidence of linkage on chromosome 10q22. Am. J. Hum. Genet 73, 601-611 (2003).

27. Faraone, S. V. et al. Genome scan of Han Chinese schizophrenia families from Taiwan: confirmation of linkage to 10q22.3. Am. J. Psychiatry 163, 1760-1766 (2006).

28. Balciuniene, J. et al. Recurrent 10q22-q23 deletions: a genomic disorder on 10q associated with cognitive and behavioral abnormalities. Am. J. Hum. Genet. 80, 938-947 (2007).

29. Morar, B. et al. Neuregulin 3 (NRG3) as a susceptibility gene in a schizophrenia subtype with florid delusions and relatively spared cognition. Mol. Psychiatn 16, 860-866 (2011).

30. Lee, M. et al. Genome-wide association study for the interaction between BMR and BMI in obese Korean women including overweight. Nutr. Res. Pract. 10, 115-124 (2016)

31. Rao, W. et al. Association between forkhead-box P2 gene polymorphism and clinical symptoms in chronic schizophrenia in a Chinese population. J. Neural Transm. Nienna) 124, 891-897 (2017).

32. Hui, L. et al. Association between DbetaH 5'-insertion/deletion polymorphism and cognition in patients with chronic schizophrenia. J. Clin. Psychiatry 77, 379-385 (2016). 
33. Xiu, M. H. et al. Contribution of $I L-10$ and its -592 AVC polymorphism to cognitive functions in first-episode drug-naive schizophrenia. Brain Behav. Immun. 57, 116-124 (2016).

34. Deakin, I. H. et al. Altered hippocampal gene expression and structure in transgenic mice overexpressing neuregulin 1 (Nrg1) type I. Transl. Psychiatry 8 , 229 (2018).

35. Lang, $X$. et al. FOXP2 contributes to the cognitive impairment in chronic patients with schizophrenia. Aging (Albany NY) 11, 6440-6448 (2019).

36. Meier, S. et al. Neuregulin 3 is associated with attention deficits in schizophrenia and bipolar disorder. Int J. Neuropsychopharmacol. 16, 549-556 (2013).

37. Tost, $\mathrm{H}$. et al. Effects of neuregulin 3 genotype on human prefrontal cortex physiology. J. Neurosci. 34, 1051-1056 (2014).

38. Li, Z. et al. NRG3 contributes to cognitive deficits in chronic patients with schizophrenia. Schizophr. Res. (2019). pii: S0920-9964(19)30495-5. https://doi. org/10.1016/j.schres.2019.10.060. [Epub ahead of print].

39. Paterson, C. \& Law, A. J. Transient overexposure of neuregulin 3 during early postnatal development impacts selective behaviors in adulthood. PLOS ONE $\mathbf{9}$, e104172 (2014)

40. Loos, M. et al. Neuregulin-3 in the mouse medial prefrontal cortex regulates impulsive action. Biol. Psychiatry 76, 648-655 (2014).

41. Mei, L. \& Nave, K. A. Neuregulin-ERBB signaling in the nervous system and neuropsychiatric diseases. Neuron 83, 27-49 (2014).

42. Bartolini, G. et al. Neuregulin 3 mediates cortical plate invasion and laminar allocation of GABAergic interneurons. Cell Rep. 18, 1157-1170 (2017).

43. Lewis, D. A., Hashimoto, T. \& Volk, D. W. Cortical inhibitory neurons and schizophrenia. Nat. Rev. Neurosci. 6, 312-324 (2005).

44. Kesby, J. P., Eyles, D. W., McGrath, J. J. \& Scott, J. G. Dopamine, psychosis and schizophrenia: the widening gap between basic and clinical neuroscience. Transl. Psychiatry 8, 30 (2018)

45. Ott, T. \& Nieder, A. Dopamine and cognitive control in prefrontal cortex. Trends Cogn. Sci. 23, 213-234 (2019).

46. Duncan, N. W., Wiebking, C. \& Northoff, G. Associations of regional GABA and glutamate with intrinsic and extrinsic neural activity in humans-a review of multimodal imaging studies. Neurosci. Biobehav. Rev. 47 36-52 (2014).
47. Tan, W. et al. Molecular cloning of a brain-specific, developmentally regulated neuregulin 1 (NRG1) isoform and identification of a functional promoter variant associated with schizophrenia. J. Biol. Chem. 282, 24343-24351 (2007).

48. Kao, W. T. et al. Common genetic variation in Neuregulin 3 (NRG3) influences risk for schizophrenia and impacts NRG3 expression in human brain. Proc. Natl Acad. Sci. USA 107, 15619-15624 (2010).

49. Paterson, C. et al. Temporal, diagnostic, and tissue-specific regulation of NRG3 isoform expression in human brain development and affective disorders. Am. J. Psychiatry 174, 256-265 (2017).

50. Olivo, G., Gour, S. \& Schioth, H. B. Low neuroticism and cognitive performance are differently associated to overweight and obesity: A cross-sectional and longitudinal UK Biobank study. Psychoneuroendocrinology 101, 167-174 (2018).

51. Qizilbash, N. et al. BMl and risk of dementia in two million people over two decades: a retrospective cohort study. Lancet Diabetes Endocrinol. 3, 431-436 (2015).

52. Kimhy, D. et al. Aerobic fitness and body mass index in individuals with schizophrenia: Implications for neurocognition and daily functioning. Psychiatry Res. 220, 784-791 (2014).

53. Hidese, S. et al. Relationship of handgrip strength and body mass index with cognitive function in patients with schizophrenia. Front Psychiatry 9, 156 (2018).

54. Guenard, F. et al. Genome-wide association study of dietary pattern scores. Nutrients. 9, 649 (2017).

55. Bray, G. A. Obesity is a chronic, relapsing neurochemical disease. Int J. Obes. Relat. Metab. Disord. 28, 34-38 (2004).

56. Funahashi, H., Yada, T., Suzuki, R. \& Shioda, S. Distribution, function, and properties of leptin receptors in the brain. Int Rev. Cytol. 224, 1-27 (2003).

57. Holden, K. F. et al. Serum leptin level and cognition in the elderly: findings from the Health ABC Study. Neurobiol. Aging 30, 1483-1489 (2009).

58. Li, X. L. et al. Impairment of long-term potentiation and spatial memory in leptin receptor-deficient rodents. Neuroscience 113, 607-615 (2002).

59. Shanley, L. J., Irving, A. J. \& Harvey, J. Leptin enhances NMDA receptor function and modulates hippocampal synaptic plasticity. J. Neurosci. 21, RC186 (2001).

60. Harvey, P. D., Docherty, N. M., Serper, M. R. \& Rasmussen, M. Cognitive deficits and thought disorder: II. An 8-month followup study. Schizophr. Bull. 16 147-156 (1990). 